\title{
Not All Wounds Are Visible- Covid -19 And Mental Health
}

\begin{abstract}
This article reviews the effect on mental health during noble covid-19. This article also reviews the mental health during infection outbreak, Psychological impact of quarantine, During the covid-19 pandemic, mental health issues faced by health care workers.
\end{abstract}

Keywords-Mental health, covid-19, Quarantine

\section{Introduction:}

Patients with 2019-nCoV (new coronavirus disease) confirmed or suspected may fear the effects of infection with a potentially lethal new virus and those in quarantine may feel fatigue, isolation and frustration. In addition, signs of illness, such as fever, hypoxia and cough, and adverse treatment results, such as corticosteroid insomnia, can contribute to worsening anxiety and mental distress.[1]

Coronavirus disease (COVID-19) has taken the world away by storm, to say the least. About one third of the world's population is under lockdown and they have announced a pandemic. The number of cases and deaths associated with COVID-19 are rising every day. It has been documented that the COVID-19 infection is primarily associated with respiratory symptoms, with deaths due to acute respiratory distress syndrome. From this viewpoint, COVID-19 is a medical emergency.[2]

Widespread infectious disease outbreaks, such as COVID-19, are associated with psychological trauma and mental health effects. Psychiatrists around the world should be aware of these manifestations, their correlations and strategies for managing them that encompass both the needs of specific populations and the precautionary measures needed to control COVID-19 spread. Researchers will also be mindful of the lacunae in the current literature, need to be filled in by extensive clinical practice and study over time.[11]

\begin{tabular}{|l|c|}
\hline \multicolumn{3}{|c|}{ Access this article online } \\
\hline Website: & Quick Response Code \\
www.ujds.in & \\
DOI: & https://doi.org/10.21276/ujds.2020.6.3.23
\end{tabular}

\section{Mental Health During A Pandemic:}

Pandemics can have a significant impact on infected patients, quarantined individuals, social support systems, and healthcare professionals. In the case of patients who are discovered or suspected to have the infection, an overwhelming sense of dread grows for the severity of the effects of the potentially deadly virus. The sensation of fear can also be infectious, because it could lead to anxiety among family friends, caretaker and even health staff, leading to patient isolation. The fear may be so devastating that getting a fever or flu can always be connected to COVID-19, just like a person from Chittoor, India's Andhra Pradesh state who decided to committed suicide. He surfed through the media surrounding COVID-19 and was persuaded that he was diagnosed with virus. Also after repeated attempts by members of his family and villagers trying to persuade him, due to fear of infection he kept isolating himself from members of his family; his conviction remained strong. Individuals in quarantine can experience anxiety, isolation, and depressive symptoms due to panic of the fatal virus and being away from close ones.[3]

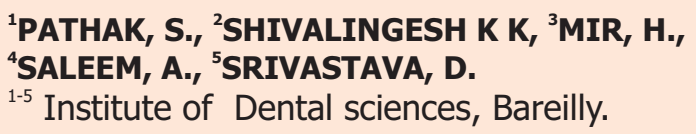

Address for Corresponding : Dr. Swati Pathak Institute of Dental sciences, Bareilly.

Email : swati.anatomy@gmail.com

Received : 3 Nov. 2020, Published : 31 Dec. 2020

How to cite this article: Pathak, S., Shivalingesh K K, Mir, H., Saleem, A., \& Srivastava, D. (2021). Not all wounds are visible- COVID -19 and mental health . UNIVERSITY JOURNAL OF DENTAL SCIENCES, 6(3). 
During the past, outbreaks of infections such as Acquired Immunodeficiency Syndrome ( AIDS), Severe Acute Respiratory Syndrome (SARS) and Ebola have affected people's minds, causing fear and behaviour related to them.[4]

Similarly, the 2014-2016 Ebola epidemic reported high rates of psychological problems, such as posttraumatic stress disorder (PTSD), not only among patients, but also among their caregivers, medical professionals, and burial authorities.[5]

\section{Psychological impact of quarantine:}

Quarantine is about isolating and limiting people's movement who was possibly exposed to an infectious disease to decide whether they are unwell, thereby reducing the chance of infection to others. The definition varies from isolation, that is disconnection of persons afflicted with a contagious disease from non-sick persons. However these two terms are sometimes used interchangeably. This term quarantine was first used in Venice, Italy, in 1127 and was commonly used in reaction to the Black Death although it wasn't until 300 years later that Britain finally began to impose quarantine during the plague. Quarantine was most recently used in coronavirus disease outbreak 2019 (COVID-19). This epidemic saw entire cities in China essentially put under mass quarantine, while several thousands of foreign nationals returning from China were forced to separate themselves at their home or in state-run establishment.[6]

Although isolation helps to achieve the goal of reducing infections, reduced access to family, friends and other systems of social support cause loneliness which results in mental conditions including anxiety and depression.[7]

During these stressful circumstances, the government, hospitals, educational institutions, organizations and even individuals involved need to evaluate psychological intervention and

take the appropriate steps. In addition to educating individuals to remain isolated, educating them and preparing them to deal with the mental health issues that they may endure over the period is essential.[7]

Mental health issues faced by health care workers in the time of pandemic covid-19
The covid-19 pandemic is likely to place healthcare professionals around the world in an unusual situation, with tough decisions to take and operate under severe pressure. This may include how to distribute scarce resources to patients who are equally in need, to balance their mental and physical health needs with those of patients, to stabilize your interest and responsibility with those of your close ones, and care for all chronically unwell patients with limited or insufficient resources. This can cause discomfort from mental health problems.[8]

All health care workers must be prepared for the moral problems they face during the covid-19 pandemic. We know that training workers adequately for the job and the related pressures decreases the risk of mental health issues. Do not give them wrong surety, but a thorough and frank evaluation of what they face, delivered in plain English and without euphemisms. When reality bites, doing anything else could add to the feelings of anger.[8]

\section{Crucial function for psychiatrists:}

Psychiatrists may play a crucial role in promoting the health of affected and their families, health care at-risk workers and the public. Psychiatrists are in fact in a unique position to provide a holistic viewpoint to strengthen the disease awareness, behaviours and practices as well as resolve the prevalent anxiety and apprehension. Here are few areas where the psychiatrists could intervene:

\section{Educating on the rising psychological adverse effects:}

Infections can trigger an Different social and behavioral consequences. Patients may be informed about common reactions to stress such as insomnia, panic attacks, health anxiety, fear of illness or increased use of substances. Children and adolescents may display varied emotional responses such as irritability, alienation or hostility that may be misinterpreted as 'regressive' behaviours. It is necessary to notify the public to general measures to counter stress such as sleep hygiene, scheduling of activities, exercising, social connections, avoiding forwards in social media and relaxation techniques. They must told about the available support outlets and websites.[9]

\section{Encouraging behaviours that promote health:}

The general public must be directed at authentic sources of 
information, such as the WHO, CDC, etc., which can reduce distress. We must be vigilant in taking the requisite precautions and in case of an epidemic, prepare measures ahead. You do ought to learn how their place of work / school wants to deal with it, because it has a calming impact on defending themselves. It is also suggested that they minimize access to media-related disinformation during times of crisis, that makes a significant contribution to fear. Collective duty may be to check the accuracy of any outbreak-related information.[9]

\section{Integrating the healthcare facilities available:}

Psychiatrists can play a significant role in improving treatment quality through interdisciplinary cooperation and education in order to keep the other medical staff open to the experiences of mental wellbeing and provide rapid intervention at the times of stress. Non-infected individuals should get ample confidence and hope to overcome with their anxiety. Focusing early on their mental health could improve their quality of life.

It is important to integrate Public programs to identify health threats and coordination during emergencies. Early detection of a pandemic's behavioural consequences helps to assess community behaviours and responses to resolve it.[9]

\section{Ensures problem-solving:}

Potential instability is one of the main life-affecting variables in new infections. This should be motivated to take selfefficacy steps to avoid hopelessness and social isolation. Large gatherings and excessive travel are typically prohibited, thereby allowing for alternate types of social connections. People need to know the steps of self-relaxation and self-care for themselves and their friends.[9]

\section{Patients, their families and health care professionals are empowered:}

The consequences of isolation and quarantine also include anxiety, irritation, afraid of infection, sleeplessness and irritability. Such short-term symptoms may also lead to a persistent post-traumatic stress syndrome and adjustment disorders. Consumption of substances has continued to increase. Ensuring sufficient dignity and resources for patients in isolation is important. Early supporting services and fostering social ties to them help alleviate loneliness. To that their fear of confusion, their families must be kept well informed by detailed, updated and appropriate information.[9]

\section{The healthcare provider self-care:}

Constant infectious exposure induces fear of infection and spreading the infection to loved ones. Chronic anxiety, shame, helplessness, loneliness and insomnia can interfere with work. It's important to have daily meals, sleep and workbreaks. Constructive peer engagement, supportive therapy and early mental health programmes contribute a great deal to reducing absenteeism and increasing their standard of treatment for themselves and the patients themselves. Effective detection of depression and prompt psychological interventions can not only avoid pandemic crisis but also help to curtail its spread.[9]

How do individuals build optimal frameworks for a mentally healthy life which works for them in the wake of COVID-19 and social and physical distance?

In the wake of COVID-19 the optimal structure of a mentally healthy life for individuals needs to be mapped. The arrangement varies according to the context and individual circumstances. Changes in habits in sleep and lifestyle impact our mental health and reaction to stress. It is of paramount importance to understand the effective, individualized approaches to coping in such a situation. Under particularly stressful circumstances, the social and personal resources (e.g. seeing the family and getting enough sleep) available to individuals can be important resilience-related factors to mitigate mental health problems. We need work to foster productive social capital, resilience and unselfishness. Operating from home, work cuts, and social and physical distancing suddenly disrupted several significant social opportunities for physical and psychological wellbeing. Researching the mental health aspect of online life is significant, and exploring how shifts in engagement with gaming and online media could influence initiatives aimed at improving mental health. We need to learn quickly from effective current approaches for sustaining and developing social capital and resilience, and fostering good mental well being in different communities.[10]

\section{References:}

1. Carvalho PM, M.M. Moreira, M.N.A. de Oliveira, J.M.M. Landim, M.L.R. Neto The psychiatric impact of the novel coronavirus outbreak PsychiatryRes., 286 
$\left(\begin{array}{llll}2 & 0 & 2 & 0\end{array}\right), \quad$ A r t i c l e 1129902 , 10.1016/j.psychres.2020.112902Google Scholar.

2. S. Grover, D. Dua, S. Sahoo, A. Mehra, R. Nehra, S. Chakrabarti Why all COVID-19 hospitals should have mental health professionals: the importance of mental health in a worldwide crisis! Asian J. Psychiatr., 51 (2020), Article 102147, 10.1016/j.ajp.2020.102147

3. Joseph SJ, P G, Bhandari SS, Dutta S. How the novel coronavirus(COVID-19) could have a quivering impact on mental health? Open JPsychiatry Allied Sci. 2020 Mar 27.

4. Bali S, Stewart KA, Pate MA. Long shadow of fear in an epidemic: fearonomic effects of Ebola on the private sector in Nigeria. BMJ Glob Health. 2016; 1:e000111.

5. O'Leary A, Jalloh MF, Neria Y. Fear and culture: contextualisingmental health impact of the 2014-2016 Ebola epidemic in WestAfrica. BMJ Glob Health. 2018;3:e000924.

6. SK Brooks, RK Webster, LE Smith, et al.The psychological impact of quarantine and how to reduce it: rapid review of the evidence Lancet, 395 (2020), pp. 912920.

7. Hiremath P, SuhasKowshik CS, Manjunath M, Shettar M. COVID-19: Impact of lockdown on mental health and tips to overcome. Asian J Psychiatr2020 Apr 10;51:102088.

8. Greenberg, N, Docherty, M., Gnanapragasam, S. , \&Wessely, S. (2020).Managing mental health challenges faced by healthcare workers during covid-19 pandemic. The BMJ, 368 10.1136/bmj.m1211.

9. D. Banerjee, The COVID-19 outbreak: crucial role the psychiatrists can play Asian J. Psychiatry (2020), 10.1016/j.ajp.2020.102014102014.

10. Holmes EA O'Connor RC Perry VH et al. Multidisciplinary research priorities for the COVID-19 pandemic: a call for action for mental health science. Lancet Psychiatry. 2020; (published online April 15.)

11. R.P. Rajkumar COVID-19 and mental health: a review of the existing literature Asian J. Psychiat., 52 (2020), Article 102066, 10.1016/j.ajp.2020.102066 